\title{
THE TIME COURSES OF VISUAL MISLOCALIZATION AND OF EXTRARETINAL EYE POSITION SIGNALS AT THE TIME OF VERTICAL SACCADES
}

\author{
HITOSH HONDA \\ Department of Psychology, Faculty of Humanities, Niigata University, Niigata 950-21, Japan
}

(Received 14 November 1990; in revised form 27 February 1991)

\begin{abstract}
The time course of perceptual mislocalization at the time of vertical saccades was compared with that reported previously for horizontal saccades. Despite the difference in the oculomotor system involved in generating these saccades with different directions, the time course of the perceptual mislocalization and that of the extraretinal eye position signal estimated on the basis of visual mislocalization were the same between vertical and horizontal saccades. The results are discussed in relation to the outflow theory of subtraction, and it is argued that there is a common neural center for producing extraretinal eye position signals for both vertical and horizontal saccades.
\end{abstract}

Vertical saccade Visual stability Eye position signal Position constancy

\section{INTRODUCTION}

When we change the direction of our gaze in the visual field, the stationary objects do not normally appear to move, in spite of the changed positions of their images on our retinas. This phenomenon is well known as "position constancy". A classical and most pervading explanation for this phenomenon is the subtraction theory that was suggested by Helmholtz (1866). The subtraction theory explains that the shift of visual images on the retina at the time of eye movements is canceled by an extraretinal eye position signal (EEPS), resulting in visual stability.

Many psychophysical experiments, however, have failed in showing visual stability at the time of a saccadic eye movement. In typical experiments, a visual target is flashed in the dark (Honda, 1989; Matin, Matin \& Pearce, 1969; Matin, Matin \& Pola, 1970) or on an illuminated background (Bischof \& Kramer, 1968; Mateeff, 1978; O'Regan, 1984) when a subject makes a saccade, and the subject is asked to indicate the apparent position of the target. The results have been that the targets are seen at different positions from their actual positions; i.e. visual stability breaks down.

The detailed time course of visual mislocalization (visual instability) was recently reported by Honda (1990) who showed that when subjects made a horizontal saccade a visual target flashed immediately before or at the beginning of the saccade was mislocalized in the same direction as the saccade, whereas when the target was flashed at the end or immediately after the saccade the subjects mislocalized it in the opposite direction to the saccade. According to the subtraction theory, this finding suggests that the EEPS did not cancel the movement of the target's image on the retina, probably because the time course of the EEPS did not coincide with that of actual eye movement.

The present study was conducted to investigate the time courses of mislocalization and of EEPS at the time of vertical saccades. It should be noted that, in most studies reported so far, visual mislocalization has been investigated concerning horizontal saccades exclusively. However, horizontal and vertical saccades are generated via different oculomotor systems, and different extraocular muscles are involved in producing horizontal and vertical saccades. Several lines of evidence suggest that the paramedian pontine reticular formation (PPRF) in the brain stem is responsible for producing eye movements in the horizontal plane (Cohen \& Henn, 1972; Cohen \& Komatsuzaki, 1972; Keller, 1974). Keller (1974), for example, reported that most burst cells in the PPRF activated only when horizontal saccades were made. On the other hand, it is well known that cell activity in the 
mesencephalic reticular formation (MRF) is indeed related to vertical eye movements; burst cells in the MRF fire when vertical saccades are made (Büttner, Büttner-Ennever \& Henn, 1977; King \& Fuchs, 1979). Therefore, there is a possibility that the findings obtained for horizontal saccades do not necessarily hold true for vertical saccades. However, despite these differences in saccade generation between horizontal and vertical direction, no attempt has been made to explore the mislocalization at the time of vertical saccade. In this study, therefore, I asked the subjects to make vertical saccade, and compared the results with those reported for horizontal saccades.

\section{METHOD}

\section{Subjects}

Two subjects participated in this experiment. Subject HH was the author and subject NH was a university student who had no experience in eye-movement experiments and, therefore, had no knowledge about the purpose of this experiment.

\section{Eye movement recording}

Vertical movements of the subject's right eye were monitored by a photoelectric method. The subject's right eye was illuminated by an i.r. light-emitting diode (Toshiba, TLN101), and the reflected light from the two points of the lower limbus (iris-sclera boundaries in 4 o'clock and 8 o'clock positions) was collected by a pair of fiber optic bundles. A phototransistor
(Toshiba, TPS601) was attached to the end of each fiber optic bundle, and vertical eye movements were recorded by adding the two phototransistor outputs together. The accuracy of this apparatus was $0.5 \mathrm{deg}$ in visual angle, and the frequency characteristic curve was almost linear up to $300 \mathrm{~Hz}$.

\section{Procedure}

The experiment was conducted in total darkness. The subject was seated with the head fixed by a dental bite board and a forehead rest. On each trial, a buzzer warning signal was given, and then a fixation point (red LED, $0.3 \mathrm{deg}$ in dia. and $20 \mathrm{~cd} / \mathrm{m}^{2}$ in luminance) was presented for $1-1.8 \mathrm{sec}$ at the position of $4 \mathrm{deg}$ below the center of the visual field (Fig. 1). The subject was asked to binocularly keep watching the fixation point. At the offset of the fixation point, a visual cue for saccades was presented for $20 \mathrm{msec}$, at the position $4 \mathrm{deg}$ above the center of the visual field. The visual cue consisted of two horizontally arranged rectangular red LEDs. Each LED $\left(10 \mathrm{~cd} / \mathrm{m}^{2}\right)$ was $0.3 \mathrm{deg}$ in height and $0.1 \mathrm{deg}$ in width. The distance between the centers of each LED was $0.4 \mathrm{deg}$. The subject was asked to make a vertical saccade (primary saccade) toward the visual cue. Because the duration of the visual cue was too short $(20 \mathrm{msec})$, it disappeared before the beginning of the saccade.

At various points in time before, during, or after the saccade, a visual target (yellow LED, $0.3 \mathrm{deg}$ in $\mathrm{dia} ., 40 \mathrm{~cd} / \mathrm{m}^{2}$ ) for visual localization was flashed for $2 \mathrm{msec}$. To present the target

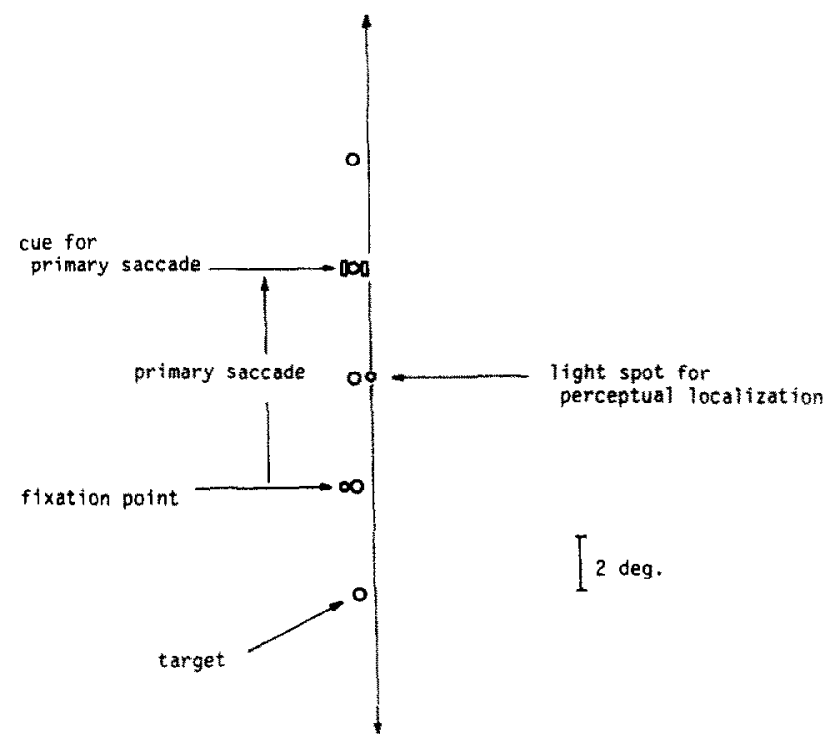

Fig. 1. Relative locations of the fixation point, the visual cue for the primary saccade, 5 positions of the target, and the adjustable light spot for perceptual localization. 
during or after the saccade, the output from the eye movement monitor apparatus was fed into a differential circuit that triggered the onset of the targets. Targets before the saccade were presented by pre-setting a shorter time interval than the normal saccade latencies between the target and the visual cue for eliciting the saccade. The position of the target was randomly selected from 5 positions, $-4,0,+4,+8$, and $+12 \mathrm{deg}$, where the minus and the plus signs indicate the downward and upward directions from the fixation point, respectively. The subject was asked to move the eyes to where the target had disappeared and to maintain fixation. About $1.4 \mathrm{sec}$ after the presentation of the target, a probe stimulus (a spot of laser beam, $0.2 \mathrm{deg}$ in dia., about $40 \mathrm{~cd} / \mathrm{m}^{2}$ ) was presented for $5 \mathrm{sec}$. The subject could move the vertical position of the probe by moving a knob by his right hand. The subject reported the apparent position of the target by moving the probe to that position.

In addition to the saccade condition described above, accuracy of perceptual judgement was also examined in a control condition in which the target was presented when the eye remained still. Under this condition, either the fixation point or the visual cue for eliciting a saccade was

A
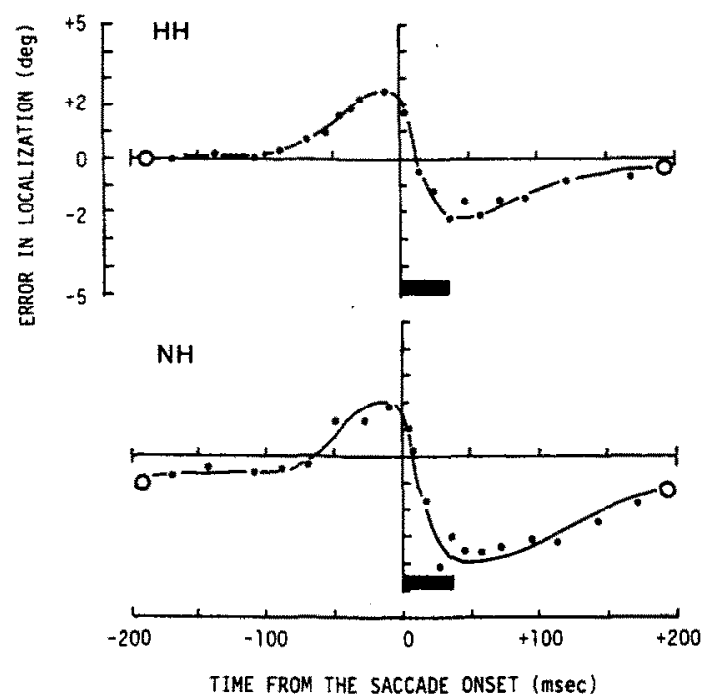

presented for $1.8 \mathrm{sec}$, and the subject was asked to keep watching these stimuli. Just after the offset of these stimuli, a target was presented for 2 msec. The subject made a saccade to the target, and reported its apparent position by moving a probe stimulus. Each subject participated in the experiment for 7-8 days. On each day, 5 sessions of 14 experimental (saccade) trials and 2 sessions of 10 control trials were given for each subject. The sessions on the first day were regarded as practice and were excluded from the data analysis.

\section{RESULTS}

\section{Primary saccade}

The subject's eye movement was analyzed by a high-speed digital storage scope (Iwatsu, DS$6121 \mathrm{~A})$. When a target was presented immediately after the presentation of the visual cue for eliciting a primary saccade, the eye sometimes moved directly to the target. In this case, therefore, a saccade to the visual cue for eliciting a primary saccade did not occur. This type of response was observed on 15 trials $(3.5 \%)$ and 32 trials $(7.5 \%)$ out of a total of 420 trials in subjects $\mathrm{HH}$ and $\mathrm{NH}$, respectively. In addition, the subjects sometimes made saccades with extremely short $(<50 \mathrm{msec})$ or

B

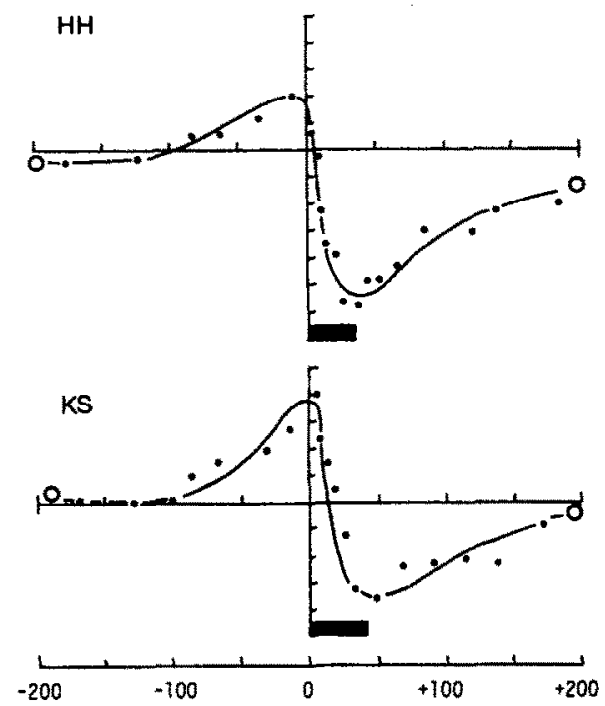

Fig. 2. (A) Time course of perceptual mislocation at the time of vertical saccades. The abscissa indicates the time interval between saccade onset and target presentation. The ordinate indicates the size of mislocalization of the targets. Plus sign in the ordinate shows mislocalization in the saccade direction, and minus sign mislocalization in the direction opposite to the saccade. Each dot in the figure represents the mean of about 10-60 trials. Open circles indicate the results on control trials in which the subjects kept watching the original fixation point (left circle) or the visual cue for eliciting a saccade (right circle). Horizontal bars show the mean duration of the saccades. (B) Examples of the time course of perceptual mislocalization at the time of horizontal saccades. [Figure redrawn from Honda (1990).] 
long ( $>300 \mathrm{msec}$ ) latencies. These expected or delayed responses were shown on 49 trials $(11.7 \%)$ in subject $\mathrm{HH}$, and on 114 trials $(27 \%)$ in subjects $\mathrm{NH}$. These trials were excluded from the following data analysis.

The means of the amplitude, the latency and the duration of the primary saccades were $8.3 \mathrm{deg}(\mathrm{SD}=1.0), 168 \mathrm{msec}(\mathrm{SD}=27)$, and $38 \mathrm{msec}(\mathrm{SD}=5.5)$, respectively, in subject $\mathrm{HH}$, and $8.1 \mathrm{deg}(\mathrm{SD}=2.3), 174 \mathrm{msec} \quad(\mathrm{SD}=31)$, and $39 \mathrm{msec}(\mathrm{SD}=8.3)$ in subject $\mathrm{NH}$.

\section{Visual mislocalization}

The discrepancy between the actual target position and the perceptually judged position was calculated on each trial, and analyzed in relation to the timing of the target presentation. Figure 2(A) shows the error in perceptual judgement as a function of the time from the saccade onset. When a target was presented immediately before or at the beginning of a saccade, the subjects mislocalized the target in the direction of the primary saccade. The mislocalization appeared from about $100 \mathrm{msec}$ before the saccade onset. At the end and immediately after the completion of the primary saccade, the targets were mislocalized in the opposite direction of the saccade. The mislocalization continued even after the end of the saccade. When targets were presented about midway through the saccade, the subjects perceptually localized the targets near the actual position. The time course of mislocalization was completely the same as that reported concerning horizontal saccades of $8^{\circ}$ in size [Fig. 2(B)]. Furthermore, the time course of perceptual mislocalization was about the same across the 5 target positions (Fig. 3).

\section{Extraretinal eye position in signal}

Next, the time course of the EEPS was calculated following the equation described by Mateeff (1978). According to the subtraction theory, the perceptually judged position of the target (perceived position: PP) will be determined on the basis of the combination of EEPS and information about the target position on the retina (retinal signal: RS). On this assumption, EEPS is

$$
\text { EEPS }=\text { PP }- \text { RS. }
$$

Figure 4(A) shows the time course of the EEPS, i.e. the point-to-point subtraction of RS from PP, together with that of actual eye movements. The RS was calculated on the basis of the eye position at the time of each target's presentation. In calculating EEPS, PPs were corrected by using the perceived positions obtained in control conditions in which targets were presented while the eye was being kept still, because the results of the control conditions indicated that there was a small indigenous error (open circles in Fig. 2). In addition, it may be necessary to take into consideration the time it takes for visual information to traverse the retina and reach the central visual system, the time the oculomotor system takes to move the eye, and the time the EEPS requires to reach the visual system. However, at the present

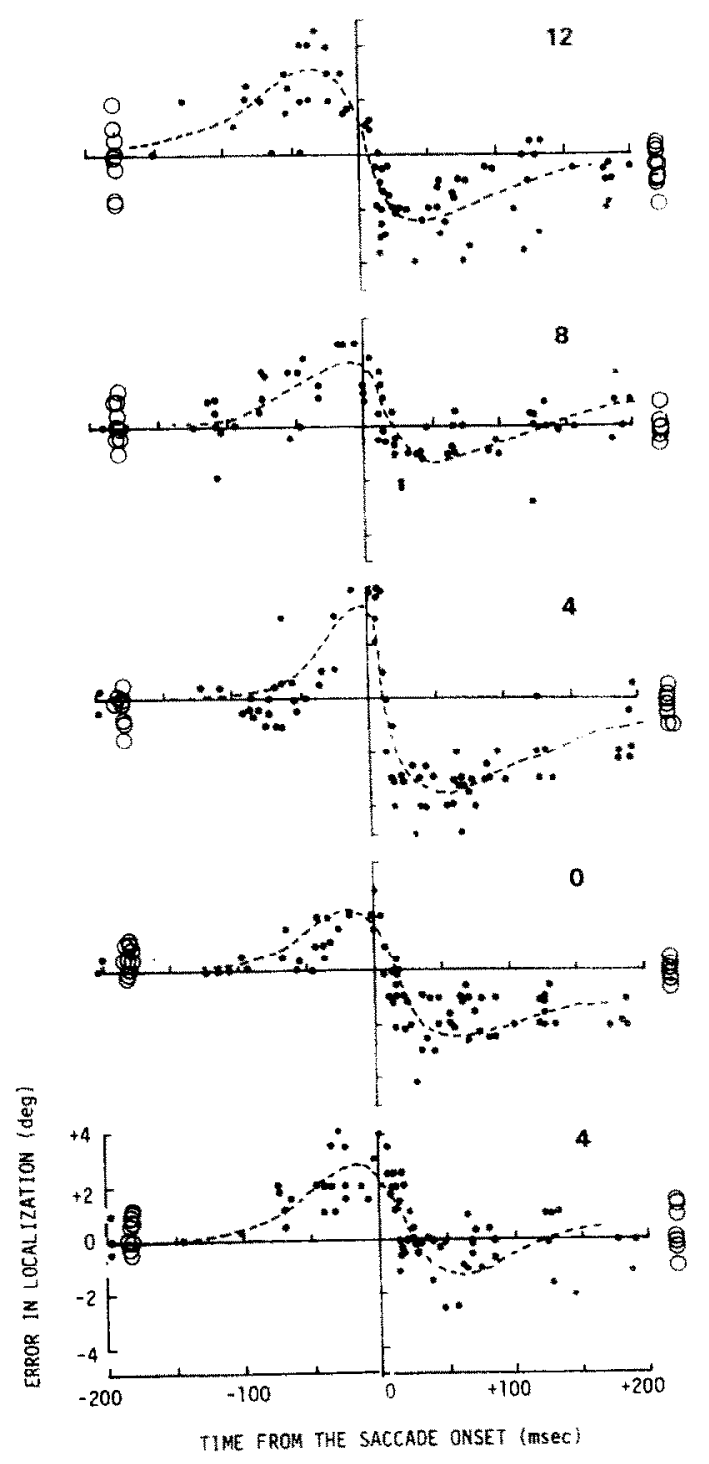

Fig. 3. Perceptually judged position plotted as a function of the time from the saccade onset, separately for the 5 actual target positions (subject $\mathrm{HH}$ ). Open circles in the figure are the positions judged on control trials. Dashed curves represent the approximate time course of perceptual error. These curves were fitted by eye based on the average errors calculated for each $20 \mathrm{msec}$ interval. 
A
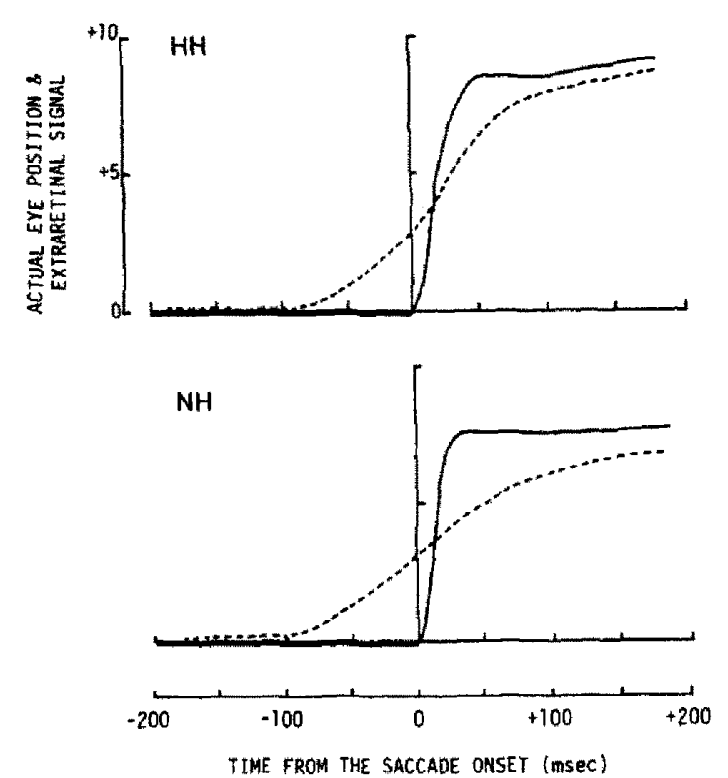

B

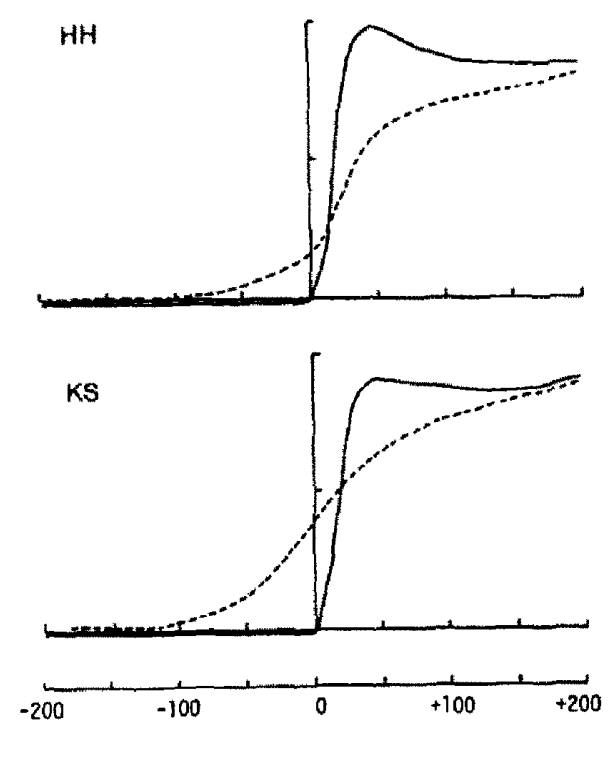

Fig. 4. (A) Comparison of the time course of actual eye movements (solid curve) and that of the EEPS (dashed curve) estimated for vertical saccades. (B) The time course of the EEPS estimated for horizontal saccades. [Figure redrawn from Honda (1990).]

time, the exact value of these processing times is not known. Therefore, we ignored the possible differences in these temporal values.

In both subjects, the time course of the estimated EEPS did not coincide with that of actual eye movement [Fig. 4(A)]. The EEPS appeared about $100 \mathrm{msec}$ before the saccade onset, but it developed so slowly that it could not catch up with the movement of the eye until more than $50 \mathrm{msec}$ after the end of the saccade. Note that there was no difference in the time course of the EEPSs between vertical and horizontal saccades [Fig. 4(B)], despite the differences in the eye muscles and the neural mechanisms for producing vertical and horizontal saccades.

\section{Secondary saccade}

The subjects were instructed, after the primary saccade, to make the secondary saccade toward the target position. When the perceptually judged position was near the position of the cue for the primary saccade, only the primary saccade occurred and the secondary saccade was omitted. On the remaining trials, subject $\mathrm{HH}$ usually made the secondary saccade. However, the destination of the secondary saccades was not the actual target's position but the perceptually judged position. On the other hand, subject NH often failed in making the secondary saccade; she made only the primary saccade, or made the secondary saccades which undershot the perceptually judged position of the target. In this case too, the goal of the secondary saccades was not the actual target position. Therefore, the results of secondary saccades conflicted with the conclusion presented by Hallett and Lightstone (1976a, b) that the eyes move toward the actual position of a test stimulus flashed during a saccade. [The issue on oculomotor response to a visual target flashed at the time of saccades was discussed elsewhere (Honda, 1990).]

\section{DISCUSSION}

\section{Perceptual mislocalization and EEPS}

The present study indicated that there was no difference in the time course of perceptual mislocalization between horizontal and vertical saccades. In addition, the extent of mislocalization observed was about the same as that reported concerning horizontal saccades (Honda, 1990). Thus, it was established that perceptual mislocalization occurs in the same way irrespective of the direction of saccadic eye movements. In other words, there is no anisotropy in visual instability at the time of saccadic eye movements.

The second important finding of this study was that the time course of EEPS estimated for vertical saccades was the same as that for horizontal saccades. This finding gives support to 
the so-called outflow theory of subtraction that EEPS originates from the central level of the oculomotor system. Or, at least, this finding suggests that EEPS originates from the level more central than the levels of eye muscles and the brain stem, because it seems more economical for the perceptual system to get the same EEPSs for vertical and horizontal saccades from the same neural center located at the central level of the oculomotor system than to get the same EEPS from the different peripheral loci of the oculomotor system, i.e. eye muscles and the brain stem.

The second support for the outflow theory comes from the finding that EEPS proceeded saccade onset by about $100 \mathrm{msec}$. No burst cells except long-lead burst cells in the brain stem fire more than $20 \mathrm{msec}$ before the onset of a saccade. In contrast, cells in the superior colliculus and the frontal eye field discharge more than $100 \mathrm{msec}$ before the onset of a visually guided saccade (Goldberg \& Bushnell, 1981; Wurtz \& Goldberg, 1972). This suggests that the hypothesized neural center for the EEPS exists at these eye-movement-related areas.

\section{Explanation for visual stability}

The present study confirmed the earlier findings that the visual target flashed at the time of a saccade was not seen at its actual position (Bischof \& Kramer, 1968; Honda, 1989, 1990; Mateeff, 1978; Matin et al., 1969, 1970; O'Regan, 1984). In addition, it was shown that the estimated time course of the EEPS did not coincide with that of actual eye position during a saccade. These findings reject the explanation for the visual stability based on the subtraction theory. In other words, the visual stability observed in our everyday life cannot be explained in terms of subtraction, because the EEPS does not provide the perceptual system with accurate information about the actual eye position during saccade. Therefore, the visual stability in a normally illuminated and structured visual field seems to be provided by mechanisms other than cancellation.

In most experiments on visual stability, a visual target is flashed in the dark or on an illuminated background at the time of a saccade. In contrast, in our everyday life visual scenery continuously exists at the same position before, during, and after a saccade. Therefore, the visual stability in our everyday life seems to be provided by a suppression mechanism such as the visual masking effect of a clear image before and after a saccade on the gray-out during the saccade (Campbell \& Wurtz, 1978). It should be noted here that the EEPS has reached the actual eye position within about $100 \mathrm{msec}$ after the end of saccades (Fig. 4). In addition to this kind of suppression mechanism. an adaptation effect to image motion during saccade (Bridgeman, 1983) may contribute to establishing visual stability.

The second possible explanation for visual stability is that the visual system has some kind of mechanism for averaging mislocalization observed around the time of a saccade onset. As shown in Fig. 2, the perceptual error immediately before and at the beginning of a saccade was in the same direction as the saccade, whereas at the end and immediately after the saccade the error was in the opposite direction to the saccade. At about the middle point of the saccade, the visual target was localized at its actual position. Therefore, the spatio-temporal average error near the time of the saccade is about the same as the actual position of the target. It seems possible that the visual system has this kind of spatio-temporal averaging mechanism. However, suppression and adaptation effects described above seem to be a more economic and efficient mechanism for visual stability than the averaging mechanism assumed to be in the visual system.

\section{REFERENCES}

Bischof, N. \& Kramer, E. (1968). Untersuchung und Uberlegungen zur Richtungswahrnehumg bei willkürlichen sakkadischen Augenbewegungen. Psychologische Forschung, 32, 185-218.

Bridgeman, B. (1983). Mechanisms of space constancy. In Hein, A. \& Jeannerod, M. (Eds), Spatially oriented behavior (pp. 263-279). New York: Springer.

Büttner, U., Büttner-Ennever, J. A. \& Henn, V. (1977). Vertical eye movement related unit activity in the rostral mecencephalic reticular formation of the alert monkey. Brain Research, 130, 239-252.

Campbell, F. W. \& Wurtz, R. H. (1978). Saccadic omission: Why we do not see a gray-out during a saccadic eye movement. Vision Research, 18, 1297-1303.

Cohen, B. \& Henn, V. (1972). Unit activity in the pontine reticular formation associated with eye movements. Brain Research, 46, 403-410.

Cohen, B. \& Komatsuzaki, A. (1972). Eye movements induced by stimulation of the pontine reticular formation. Evidence for integration in oculomotor pathways. Experimental Neurology, 36, 101-117.

Goldberg, M. E. \& Bushnell, M. C. (1981). Behavioral enhancement of visual responses in monkey cerebral cortex. II. Modulation in frontal eye fields specifically related to saccades. Journal of Neurophysiology, 46, 773-787. 
Hallett, P. E. \& Lightstone, A. D. (1976a). Saccadic eye movements towards stimuli triggered by prior saccades. Vision Research, 16, 99-106.

Hallett, P. E. \& Lightstone, A. D. (1976b). Saccadic eye movements to flashed targets. Vision Research, 16, 107-114.

von Helmholtz, H. (1866). Handbuch der physiologischen Optik. Leipzig: Voss.

Honda, H. (1989). Perceptual localization of visual stimuli flashed during saccades. Perception and Psychophysics, 45, 162-174.

Honda, H. (1990). Eye movements to a visual stimulus flashed before, during, or after a saccade. In Jeannerod, M. (Ed.), Attention and performance (Vol. 13, pp. 567-582). Hillsdale: LEA.

Keller, E. L. (1974). Participation of the medial pontine reticular formation in eye movement generation in monkey. Journal of Neurophysiology, 37, 316-332.

King, W. M. \& Fuchs, A. F. (1979). Reticular control of vertical saccadic eye movements by mesencephalic burst neurons. Journal of Neurophysiology, 42, 861-876.
Mateeff, S. (1978). Saccadic eye movements and localization of visual stimuli. Perception and Psychophysics, 24, 215-224.

Matin, L., Matin, E. \& Pearce, D. G. (1969), Visual perception of direction when voluntary saccade occur: $\mathrm{I}$. Relation of visual direction of fixation target extinguished before a saccade to a flash presented during the saccades. Perception and Psychophysics, 5, 65-80.

Matin, L., Matin, E. \& Pola, J. (1970). Visual perception of direction when voluntary saccade occur: II. Relation of visual direction of a fixation target extinguished before a saccade to a subsequent test flash presented before the saccade. Perception and Psychophysics, 8, 9-14.

O'Regan, (1984). Retinal versus extraretinal influences in flash localization during saccadic eye movements in the presence of a visible background. Perception and Psychophysics, 36, 1-14.

Wurtz, R. H. \& Goldberg, M. E. (1972). Activity of superior colliculus in behaving monkey. III. Cells discharging before eye movements. Journal of Neurophysiology, 35, $575-586$. 